\title{
Beneficios de implementar un Clúster de bases de datos Oracle EN LOS SERVICIOS CRÍTICOS DE LAS TECNOLOGÍAS DE INFORMACIÓN*
}

\author{
Francisco Martín Riccio Chávez \\ IBM del Perú. Lima, Perú
}

Recibido: 26 de agosto de 2015 / Aprobado: 4 de setiembre de 2015

\begin{abstract}
Resumen
El presente artículo proporciona un conocimiento general sobre la infraestructura de un clúster de bases de datos Oracle, resaltando sus principales beneficios para aquellas empresas que requieran brindar servicios críticos de tecnologías de información, a través de una inversión justificada. Para una mejor comprensión, se mostrará una arquitectura general de esta tecnología, así como consideraciones técnicas y experiencias que han sido recogidas como parte de las implementaciones realizadas en ambientes on-premises y off-premises. Cabe indicar que esta investigación está enfocada a la tecnología Oracle Real Application Cluster (RAC) y a Oracle Flex Cluster, donde esta última es una nueva funcionalidad que provee la base de datos Oracle en su versión 12c, que era la versión vigente cuando fue escrito este documento.
\end{abstract}

Palabras clave: Oracle RAC / Oracle Flex Cluster / alta disponibilidad

\section{Benefits of Implementing a Clustered Oracle Database on Critical Services of the Information Technology}

\section{Summary}

This scientific article provides a general knowledge about the infrastructure of a clustering Oracle databases, which highlights the primary benefits for companies that offer critical services within the information technology (IT) realm, via a justified investment. For a better understanding, the makeup of this technology will be explained. Various research examples and implementations will also be illustrated, on-premises and off-premises, by the students of the university. It should be noted that this research will be focused on the Oracle Real Application Cluster (RAC) and Oracle Flex Cluster. The final subject is a new feature that provides the Oracle database, which references $12 \mathrm{c}$ version technology, with the current date being the same date this article was written.

Key words: Oracle RAC / Oracle Flex Cluster / High Availability

* Colaboradores: Víctor Cáceres, Hugo Castro, Bryan Cuya, Miguel Lladó y Martín Ramos. 


\section{Introducción}

Uno de los desafíos más frecuentes que presenta el área de tecnologías de información (TI), por causa de las complejidades técnicas y del presupuesto anual asignado, es el de mantener servicios de negocio críticos con un tiempo de recuperación (RTO - Recovery Time Objective) ante una falla, de 0 segundos y con una arquitectura escalable.

Para cumplir un RTO de 0 segundos primero se deben considerar todos los componentes (servidores, subsistema de almacenamiento, redes y centro de cómputo) de la arquitectura que soporta el servicio crítico de TI. Con ello se calcula la disponibilidad del servicio, multiplicando la disponibilidad individual de cada componente y comparándola con la disponibilidad esperada, en este caso de 0 segundos.

Con el propósito de alcanzar una alta disponibilidad en el componente de bases de datos se cuenta con mecanismos de clúster, los cuales han sido ofrecidos durante más de una década por diferentes fabricantes de software. El tiempo de recuperación para este tipo de soluciones, al generarse una indisponibilidad con la base de datos, se encuentra medido en minutos. Asimismo, estos productos no ofrecen una arquitectura escalable al mantener básicamente un esquema activo/pasivo.

Oracle RAC es un producto disponible desde inicios del año 2000, que tuvo como antecesor a Oracle Parallel Server (OPS); permite brindar una solución con un RTO de 0 segundos en el componente de base de datos, y también de una solución escalable, ya que soporta una mayor cantidad de sesiones concurrentes en el futuro, sin afectar el rendimiento del sistema. Esto se logra, básicamente, al mantener un esquema activo/activo en cada uno de los servidores que conforman el clúster.

Oracle Flex Cluster es una nueva opción sobre la que se puede instalar Oracle RAC con nuevas ventajas competitivas, comparándola con la instalación tradicional disponible en versiones anteriores.

Oracle ofrece un marco de trabajo llamado Maximum Availability Architecture (MAA), el cual reúne las mejores prácticas recomendadas para el diseño, implementación y operación de arquitecturas de alta disponibilidad con productos Oracle, y donde Oracle RAC es uno de los pilares de esta documentación. En Oracle Help Center (2015) se puede obtener mayor información acerca del marco de trabajo Oracle MAA y del marco de trabajo MAA.

\section{Arquitectura de Oracle Flex Cluster}

La nueva arquitectura de alta disponibilidad en Oracle 12c (Oracle Flex Cluster) está constituida por dos tipos de servidores: hub y leaf (Gómez, 2013). Estos permiten incrementar el nivel de escalabilidad y disponibilidad tanto al componente de base 
de datos como a las aplicaciones desplegadas en su infraestructura. En adelante, los servidores que conforman el clúster serán referenciados a través del término 'nodo', al ser este término el especificado por la documentación oficial de Oracle. A continuación se detallan algunos conceptos importantes al respecto:

a) Nodo hub. Son servidores que están conectados físicamente al subsistema de almacenamiento compartido. Pueden prescindir de instancias ASM, pero al menos uno debe mantenerlo para acceder físicamente a dos archivos críticos: Voting File y Oracle Cluster Registry (OCR). El uso principal de estos servidores está enfocado en el despliegue de base de datos en un esquema de alta disponibilidad.

b) Nodo leaf. Estos servidores no se encuentran físicamente conectados al subsistema de almacenamiento compartido pero sí conectados mediante una red LAN a los nodos hub, con el propósito de integrarse a la solución de clúster. El objetivo de estos servidores es ofrecer un esquema de alta disponibilidad a las aplicaciones de negocio.

c) Infraestructura grid. Es un producto de Oracle encargado de proveer el programa de clúster e, igualmente, despliega al manejador de archivos Automatic Storage Management (ASM).

d) Real Application Cluster (RAC). Es una base de datos Oracle en modalidad clúster. Una base de datos no instalada como RAC es conocida como Single Instance.

e) Instancia de base de datos. Es un conjunto de estructuras de memoria y de procesos que permiten acceder a los archivos de base de datos almacenados en disco.

f) Automatic Storage Management (ASM). Es un sistema de archivos y administrador de volúmenes principalmente destinados para archivos de bases de datos Single Instance o RAC. Esta tecnología permite almacenar los archivos OCR y Voting File. Almacenar los archivos en sistemas de archivos ASM mejora en gran medida el tiempo de acceso a la información de los datos en el disco, gracias a una serie de ventajas competitivas que vienen como parte del producto. ASM es gestionado a través de una instancia llamada +ASM, cuyo objetivo es proporcionar la información necesaria a las instancias de base de datos y así estas puedan acceder a los datos almacenados en los discos en formato ASM.

g) Oracle Cluster Registry (OCR). Almacena todos los recursos que se ejecutarán en el clúster, que requieren ser monitoreados para ofrecerles una alta disponibilidad.

h) Voting File. Almacena la lista de nodos que conforman el clúster.

Para implementar Oracle Flex Cluster existen dos tecnologías importantes que se deben tener en cuenta: Flex ASM y Grid Naming Service.

a) Flex ASM. Permite que una instancia de base de datos pueda comunicarse con una instancia ASM, incluso al encontrarse en servidores distintos. Así, la base de datos ya no dependerá exclusivamente de una instancia ASM ubicada en su 
propio servidor, como ocurría en versiones anteriores, las cuales representaban un gran riesgo respecto al nivel de disponibilidad requerido.

b) Grid Naming Service (GNS). Es una configuración que ofrece flexibilidad al permitir desplegar dinámicamente más nodos en el clúster de un modo simple, a través de una asignación dinámica de IP a los nodos del clúster.

Cada nodo del clúster contará con las siguientes direcciones IP:

a) IP pública: utilizada para actividades de administración del servidor.

b) IP privada: mantiene la comunicación, consistencia y coordinación entre los nodos hub.

c) IP virtual: esta dirección IP establece tiempos muy rápidos de respuesta en el caso de que el nodo no esté disponible, y así no esperar hasta 10 minutos para obtener un mensaje de error, como ocurre con el protocolo TCP/IP tradicional. Las aplicaciones de negocio utilizarán esta IP para acceder a un nodo específico.

d) IP ASM. Servirá para establecer la comunicación entre los nodos hub y leaf. Esta IP es opcional y puede ser asumida por la IP privada.

Todos los nodos hub podrán ser accedidos a través de un solo nombre (hostname), es decir, a través de direcciones denominadas Single Client Access Name (SCAN), que se resolverán a través de tres IP bajo el algoritmo de round-robin. Estas IP serán asignadas únicamente entre nodos hub del clúster.

La cadena de conexión que utilizan las aplicaciones al conectarse a la base de datos en Oracle RAC debería utilizar el SCAN definido en el clúster como recomendación. Por otro lado, solo un servidor de los nodos hub llevará una IP adicional y servirá para ejecutar el servicio GNS previamente mencionado. Cabe resaltar que las IP pública, virtual, SCAN y GNS, serán definidas en la misma segmentación de red.

Un servicio DNS y otro DHCP se requieren como parte de la implementación, siendo este último el responsable de asignar un conjunto de direcciones IP a los servidores que conforman el clúster, las cuales serán distribuidas a los nodos a través de un servicio llamado GNS.

GNS tendrá la responsabilidad de operar como un servicio DNS dentro del clúster. El servidor DNS delegará al servicio GNS la responsabilidad de resolución interna de nombres de los nodos del clúster solicitado por una aplicación. Ejemplo: se adjunta la cadena de conexión hacia una base de datos desde una aplicación en Java, utilizando la librería JDBC: jdbc:oracle:thin@//rac-scan.gns.ulima.edu.pe:1521/PRD

La aplicación intenta conectarse al hostname rac-scan.gns.ulima.edu.pe, nombre del SCAN definido y cuyo dominio es gns.ulima.edu.pe. La resolución de nombre rac-scan solicitado por la aplicación deberá ser resuelto por el servidor DNS. Este dirigirá el requerimiento al servicio GNS, el cual devolverá las tres IP dinámicas que fueron asignadas por el servicio DHCP al iniciar los servicios del clúster. 
Identificadas las tres IP, y mediante el algoritmo round-robin, el servidor DNS proporcionará a la aplicación solo una IP y así iniciará las primeras fases internas de conexión a la base de datos. Posteriormente, se ejecutan una serie de pasos internamente por Oracle para establecer la conexión entre el cliente y el servidor de base de datos que ofrece un mejor rendimiento en ese momento.

En las figuras 1 y 2 se muestra la arquitectura en alto nivel de una solución Oracle Flex Cluster y Oracle GNS.

En la figura 3 se muestra la configuración realizada en el servidor DNS.

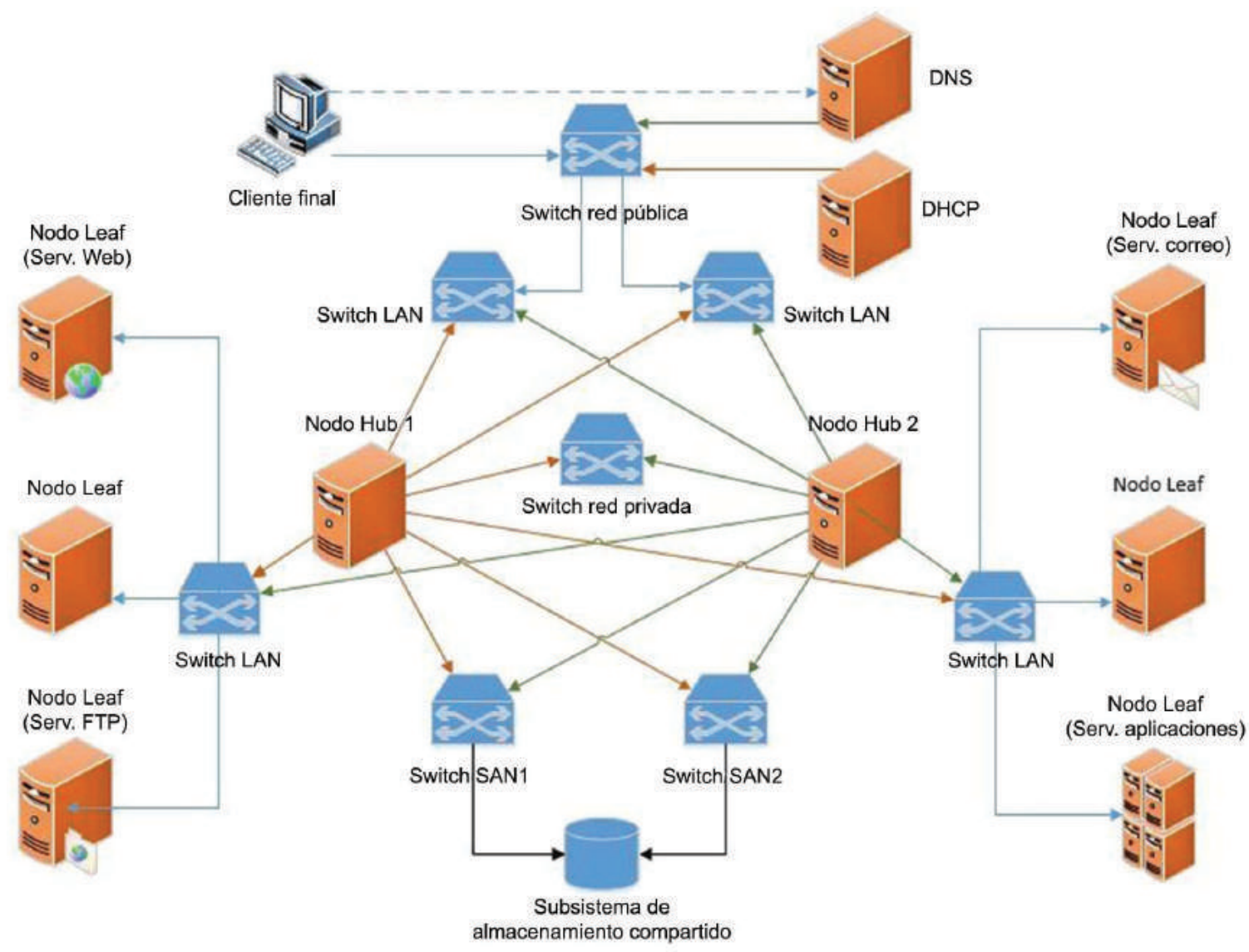

Figura 1. Arquitectura en alto nivel de una solución Oracle Flex Cluster Elaboración propia 


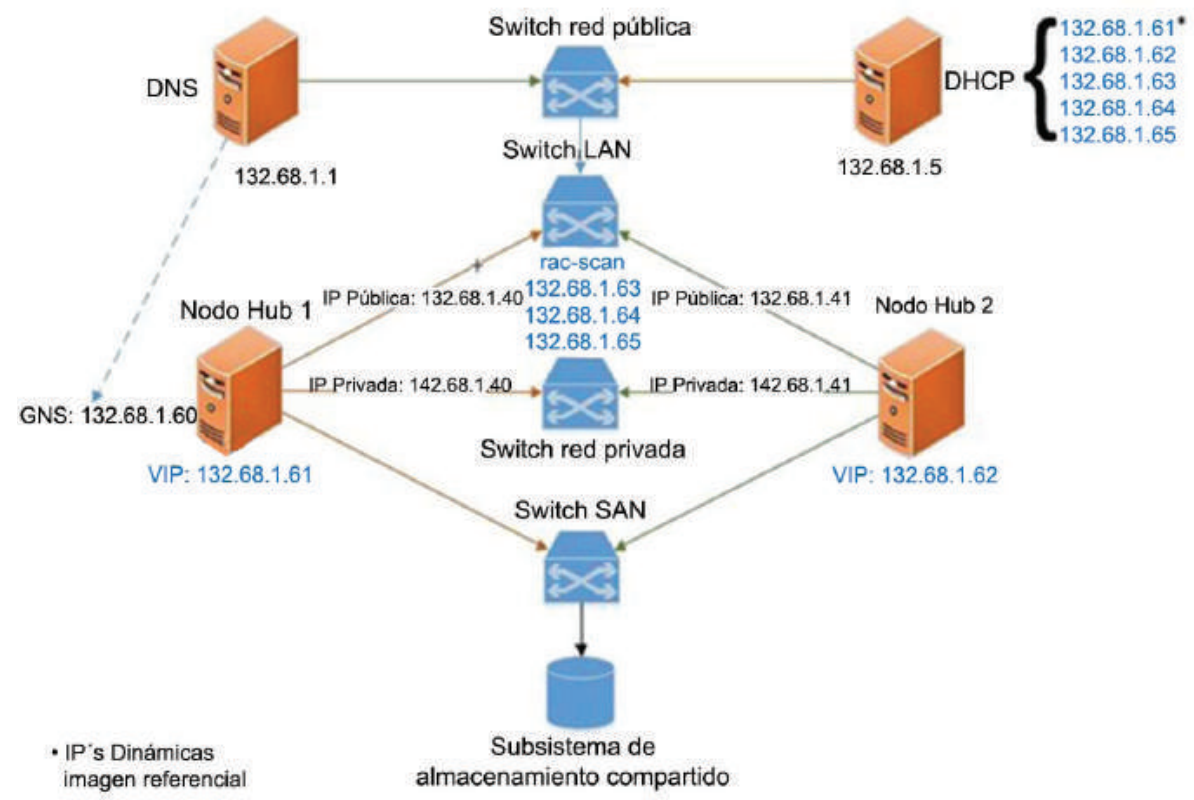

Figura 2. Arquitectura en alto nivel de una solución Oracle RAC con GNS Elaboración propia

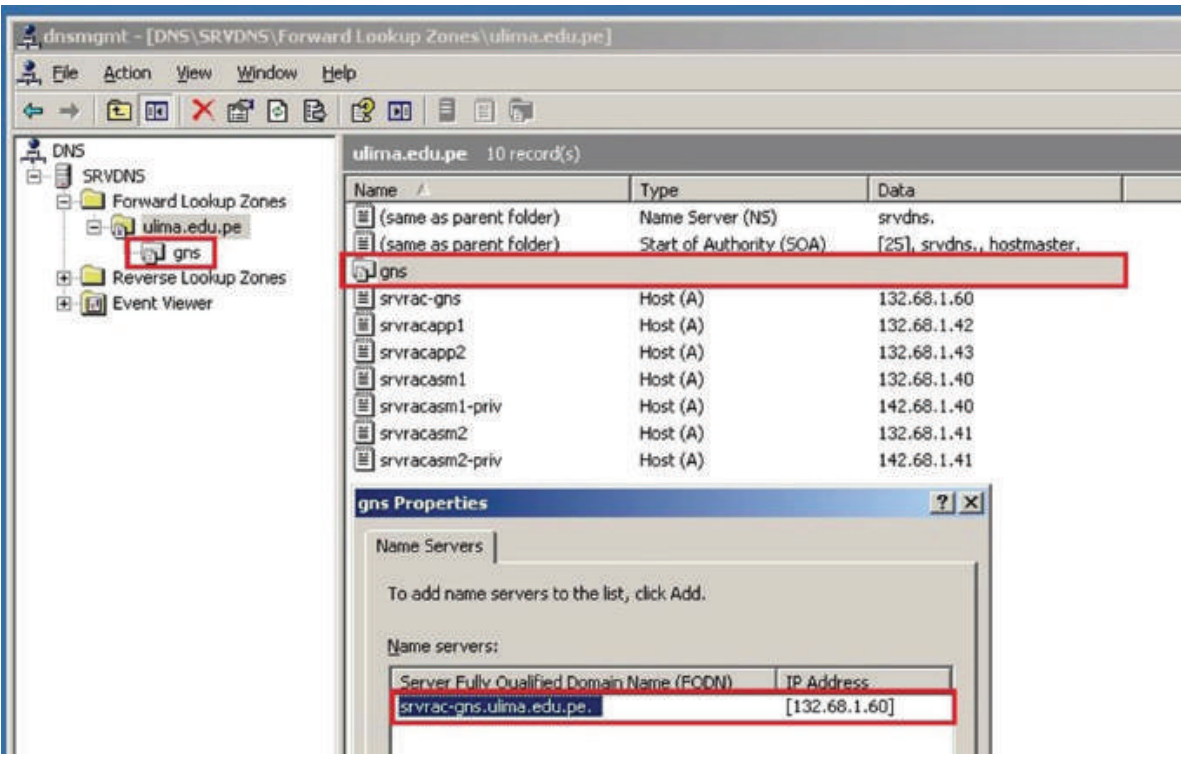

Figura 3. Configuración en el servidor DNS

Elaboración propia 
Se puede apreciar que el dominio ulima.edu.pe mantiene un subdominio llamado gns.ulima.edu.pe, que será resuelto por la IP del GNS (132.68.1.60). En la figura 4 se observa la asignación dinámica de IP virtuales y SCAN en el servidor DHCP.

\begin{tabular}{|c|c|c|c|c|c|c|}
\hline \multicolumn{7}{|l|}{ EDHCP } \\
\hline \multicolumn{7}{|l|}{ Elle Action Yew Heb } \\
\hline \multicolumn{7}{|l|}{ 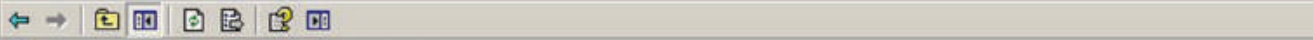 } \\
\hline \multicolumn{7}{|c|}{ A DHCP } \\
\hline - 10 srvdns [132.68.1.5] & Client IP Address & Name & Lease Explation & Type & Unique ID & Da \\
\hline 1. Address Pool & 물 132.68 .1 .61 & 132.68 .1 .61 & 28/08/2015 10:08:16 a.m. & DHCP & $636 c 7573 \ldots$ & \\
\hline Address Leases & 퉁 132.68 .1 .62 & & 28/08/2015 10:06:17 a.m. & DHCP & $636 c 7573 \ldots$ & \\
\hline + Reservations & 可 132.68 .1 .63 & & 28/08/2015 10:06:42 a.m. & DHCP & $636 \mathrm{c} 7573 \ldots$ & \\
\hline Scope Options & 尊 132.68 .1 .64 & & 28/08/2015 10:08:30 a.m. & DHCP & $73727672 \ldots$ & \\
\hline Ta Server Options & 哥 132.68 .1 .65 & & 26/08/2015 12:17:57 a.m. & DHCP & $73727672 \ldots$ & \\
\hline
\end{tabular}

Figura 4. Asignación dinámica de IP virtuales y SCAN en el servidor DHCP Elaboración propia

\section{Consideraciones en la implementación}

A continuación se detallan las experiencias recogidas por los alumnos de la Universidad de Lima al implementar una solución de Oracle RAC en dos diferentes tipos de ambiente: on-premises, Data Center de la Universidad; y off-premises, cloud computing en IBM Softlayer. Se observaron distintos aspectos y consideraciones.

\subsection{Consideraciones on-premises}

a) Validar la compatibilidad de equipos y configuraciones de infraestructura definida en la matriz de certificación de Oracle RAC.

Plataforma Linux: http://www.oracle.com/technetwork/database/clustering/ tech-generic-linux-new-086754.html

Plataforma Unix: http://www.oracle.com/technetwork/database/clústering/ tech-generic-unix-new-166583.html

Plataforma Windows:

http://www.oracle.com/technetwork/database/clústering/tech-genericwindows-new-166584.html

b) Revisar la certificación de Oracle sobre productos de virtualización: http://www.oracle.com/technetwork/database/virtualizationmatrix-172995.html

c) Cumplir con todos los requisitos previos y configuraciones, establecidos por la documentación oficial de Oracle, para una correcta instalación de Oracle RAC sobre un sistema operativo determinado:

http://docs.oracle.com/database/121/nav/portal_11.htm 
d) Mantener el correcto diseño en el subsistema de almacenamiento para asignar de manera apropiada los volúmenes de disco entre los nodos hub.

e) Elaborar una correcta distribución de IP que serán requeridas para la solución del clúster, definiendo claramente cuáles serán utilizadas para la red pública y privada. De esta manera se evita la latencia o problemas de red, que ocasionarían un fracaso en la implementación.

f) Considerar el correcto análisis de licencias requeridas, tanto para la base de datos como para Oracle RAC.

\subsection{Consideraciones off-premises}

Para la segunda implementación, realizada en un ambiente cloud computing, se deben tener las siguientes consideraciones adicionales a las previamente mencionadas en el entorno on-premises:

a) Al momento de efectuar la compra de máquinas virtuales o físicas, se recomienda revisar una por una, con la finalidad de asegurarse de que todos los servidores cuenten con las correctas VLAN definidas.

b) Dado que los servidores en cloud computing proporcionan direcciones públicas, se debe tomar las medidas adecuadas de seguridad para proteger los servidores contra ataques, ya que estos podrían ocasionar una suspensión del servicio o una mala manipulación de los datos almacenados.

c) A la fecha de edición de este artículo, ningún proveedor de cloud computing está certificado por Oracle para la implementación de Oracle RAC; por esta razón, se recomienda revisar debidamente la documentación más actual de Oracle sobre el soporte de algún proveedor, antes de iniciar una instalación. Ejemplo: la implementación de Amazon Web Service (AWS) puede revisarse en http://www.oracle.com/technetwork/topics/cloud/faq-098970.html

\section{Pruebas de desempeño y alta disponibilidad}

\subsection{Pruebas de desempeño}

Un error frecuente en el diseño de base de datos es creer que una base de datos con Oracle RAC permitirá conseguir un mayor desempeño en todas las actividades que se ejecuten; sin embargo, se demostrará lo contrario a través de un caso de prueba.

La siguiente prueba simuló 100 sesiones concurrentes, donde cada una ejecutó 500 transacciones sobre una base de datos en un entorno Oracle Flex Cluster. La base de datos contó con dos instancias en modalidad activo/activo, habiéndose comparado diversas métricas contra una base de datos sin clúster (Single Instance). 
Las características físicas de cada uno de los servidores que participaron en la prueba fueron de: 5 GB de memoria RAM y 1 vCPU, ejecutándose en un entorno Oracle Linux 6.

Se utilizó el programa HammerDB, que permitió simular un escenario basado en modelo de medición TPC-C, tal como señala la corporación TPC (2015), en un esquema cliente/servidor. En la figura 5 se aprecia que la base de datos Single Instance alcanzó un número mayor de transacciones por minuto comparada con la otra base de datos.
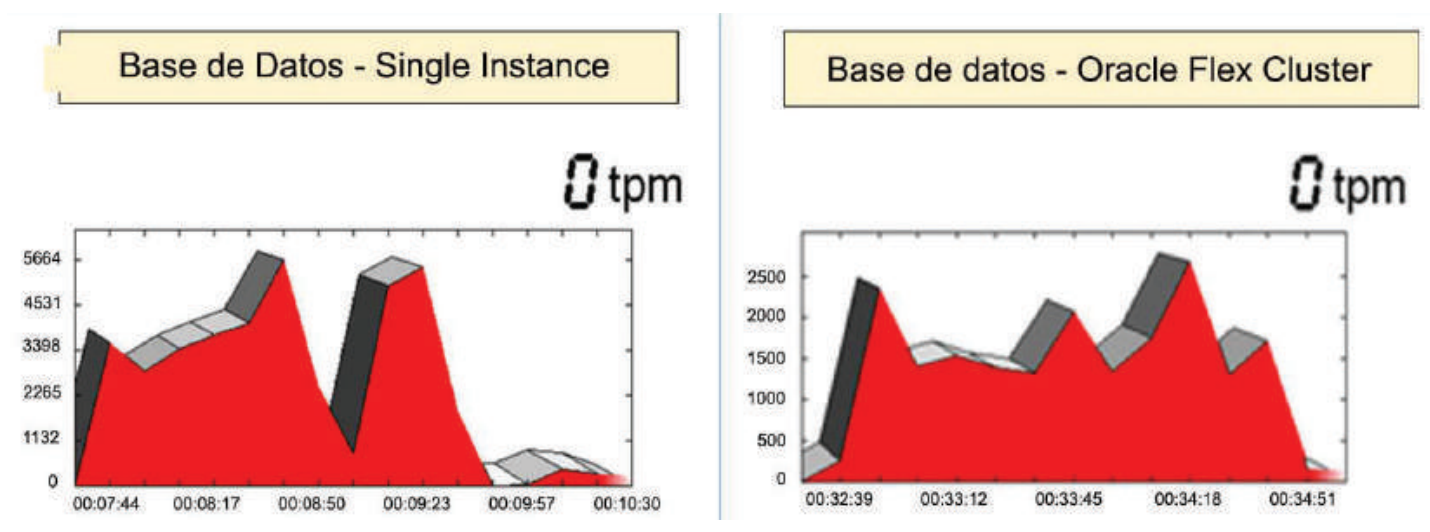

Figura 5. Comparación del número de transacciones por minuto Elaboración propia

Al revisar las métricas obtenidas por los paquetes de diagnóstico de Oracle, se observó lo siguiente:

Tabla 1

Comparación de métricas de diagnóstico

\begin{tabular}{|l|c|c|c|}
\hline & $\begin{array}{c}\text { BD Single } \\
\text { Instance }\end{array}$ & $\begin{array}{c}\text { Instancia \#1 - BD } \\
\text { Oracle Flex Cluster }\end{array}$ & $\begin{array}{c}\text { Instancia \#2 - BD } \\
\text { Oracle Flex Cluster }\end{array}$ \\
\hline $\begin{array}{l}\text { Tiempo transcurrido } \\
\text { (minutos) }\end{array}$ & 2,61 & 3,12 & 3,12 \\
\hline Tiempo de BD & 101,82 & 55,78 & 54,13 \\
\hline $\begin{array}{l}\text { Transacciones } \\
\text { por segundo }\end{array}$ & 41,8 & 17,7 & 17,5 \\
\hline
\end{tabular}

Elaboración propia 
a) La base de datos Single Instance logró una ejecución promedio de 41,8 transacciones por segundo, mientras que las dos instancias en clúster consiguieron una ejecución promedio de 35,2 (17,7 + 17,5) transacciones por segundo debido a los bloqueos que se generaron, siendo un valor ligeramente inferior.

b) Calculando la métrica Average Active Session (AAS) que permitirá medir el nivel de carga con que se encuentra la base de datos:

i. Base de datos - Single Instance $=39(101.82 / 2.61)$

ii. Base de datos - Oracle Flex Cluster (Instancia \#1) = 17.8 (55.78/3.12)

iii. Base de datos - Oracle Flex Cluster (Instancia \#2) = 17.4 (54.13/3.12)

Se puede observar que cada instancia de base de datos que conforma la solución de clúster mantiene una menor saturación comparada con la base de datos Single Instance y, por ende, puede continuar recibiendo más requerimientos. La saturación de cada instancia de base datos irá disminuyendo conforme se aumenta el número de instancias que conformen el clúster. Con este punto se demuestra que es una arquitectura escalable.

c) Uno de los beneficios disponibles con Oracle RAC es la característica de balanceo de carga, en el cual cada sesión de base de datos se reorientará al servidor que brinde un mejor servicio. Una prueba de ello es que al momento de ejecutar la prueba de desempeño con el programa HammerDB se pudo evidenciar la distribución equitativa de sesiones de base de datos entre las diferentes instancias que la conforman, de manera automática y transparente, tal como se presenta en la tabla 2:

Tabla 2

\section{Distribuciones equitativas en pruebas de desempeño}

\begin{tabular}{|c|c|}
\hline Prueba 1 & Prueba 2 \\
\hline 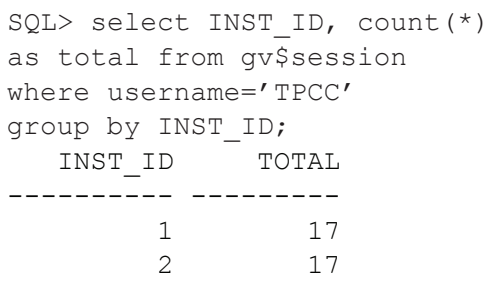 & 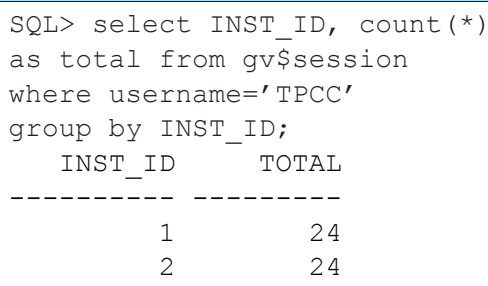 \\
\hline Prueba 3 & Prueba 4 \\
\hline 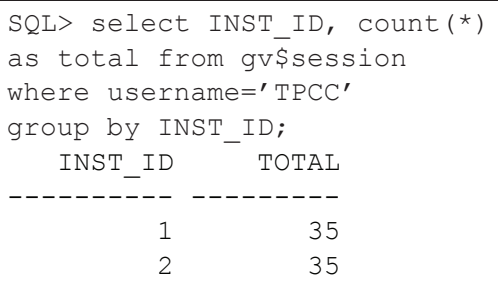 & 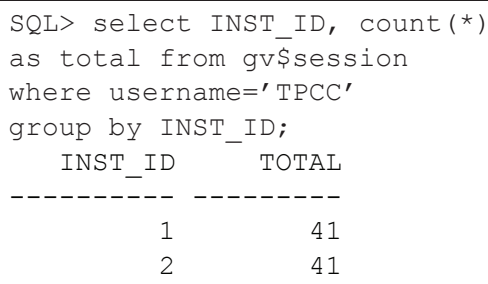 \\
\hline
\end{tabular}

\section{Elaboración propia}


Como regla general, una base de datos Oracle RAC se beneficiará de una mejora de desempeño en los escenarios detallados.

Tabla 3

Mejora de desempeño en una base de datos Oracle RAC

\begin{tabular}{|l|c|c|}
\hline \multicolumn{1}{|c|}{ Tipo de carga } & Aumento de desempeño & Escalabilidad \\
\hline Transaccional & No & Sí \\
\hline Soporte de decisiones & Sí & Sí \\
\hline Procesamiento por lotes & $\begin{array}{l}\text { Posible, si los procesos pueden } \\
\text { dividirse y ejecutarse en paralelo }\end{array}$ & Sí \\
\hline
\end{tabular}

Fuente: Womack, 2008, p. I. 12

En todos los casos siempre se conseguirá escalabilidad, tal como se demostró en la prueba previamente presentada. También se comprobó que las bases de datos con muchas actividades transaccionales no se beneficiarán de una mejora de desempeño, ya que las transacciones no son divisibles para ejecutarse mediante procesos en paralelo.

\subsection{Pruebas de alta disponibilidad}

Como parte de las pruebas de alta disponibilidad, mediante el lenguaje Java se desarrolló un programa para demostrar que, ante la caída de un servidor de base de datos, la aplicación sigue operando de manera transparente para los usuarios. La prueba de alta disponibilidad ha sido publicada en el sitio web https://www. youtube.com/watch?v=koR-9jGXxWQ

Asimismo, es importante mencionar que para desarrollar una aplicación que realmente aproveche las bondades de Oracle RAC, como el balanceo de carga, la tolerancia de fallos, etc., previamente se debe realizar configuraciones tanto en las configuraciones del clúster y base de datos como en la codificación de la aplicación. Se recomienda revisar esta documentación para un mayor detalle en http://docs. oracle.com/database/121/ADFNS/adfns_avail.htm

A continuación se listan documentaciones sobre las diversas modalidades que pudieran implementarse sobre aplicaciones.NET en un entorno Oracle RAC:

i. http://www.oracle.com/technetwork/es/articles/dotnet/transparentapplication-failover-2190706-esa.html

ii. http://www.oracle.com/technetwork/es/articles/dotnet/fast-connectionfailover-2167946-esa.html

iii. http://www.oracle.com/technetwork/es/articles/dotnet/implementandotransaction-guard-2190656-esa.html 


\section{Análisis FODA}

El análisis FODA se desarrolló desde la perspectiva de disponer de licencias Oracle RAC en la empresa para el área de TI.

Tabla 4

\section{Análisis FODA}

\begin{tabular}{|c|c|}
\hline Fortalezas & Oportunidades \\
\hline $\begin{array}{l}\text { - Oracle Flex Cluster permite integrar aplicacio- } \\
\text { nes en un esquema de alta disponibilidad en } \\
\text { múltiples nodos leaf, sin generar un costo extra } \\
\text { de licenciamiento } \\
\text { - Oracle GNS posibilita mantener un esquema } \\
\text { de configuración dinámica, donde añadir o re- } \\
\text { mover nodos del clúster está bajo la filosofía de } \\
\text { plug and play. } \\
\text { - Oracle RAC se encuentra disponible en el } \\
\text { mercado desde hace más de quince años, } \\
\text { logrando tener múltiples referencias de casos } \\
\text { de éxito en diferentes empresas por sector. }\end{array}$ & $\begin{array}{l}\text { - Arquitectura escalable, que permite incremen- } \\
\text { tar el número de nodos del clúster, para so- } \\
\text { portar la carga actual y mantener los mismos } \\
\text { niveles de tiempo de respuesta. } \\
\text { - Alta disponibilidad, que permite desplegar } \\
\text { aplicaciones que puedan ofrecer a los usuarios } \\
\text { finales una disponibilidad cercana al } 100 \text { \%. } \\
\text { - Diseñar aplicaciones que puedan aprovechar } \\
\text { Transaction Guard, el cual permite a la apli- } \\
\text { cación identificar si una transacción no pudo } \\
\text { completarse debido a una caída del servidor y } \\
\text { así enviarla nuevamente a procesar. } \\
\text { - Las licencias de Oracle RAC se amortizan en } \\
\text { un flujo de caja, lo cual brinda un ahorro en el } \\
\text { pago de impuestos. }\end{array}$ \\
\hline Debilidades & Amen \\
\hline $\begin{array}{l}\text { - Tiene aplicaciones cuyas librerías de acceso a } \\
\text { datos pueden presentar incompatibilidades } \\
\text { con Oracle RAC. } \\
\text { - El costo de mantenimiento es alto. } \\
\text { - Oracle Flex Cluster presenta problemas de } \\
\text { desempeño en un esquema de clúster exten- } \\
\text { dido, debido a que una instancia de base de } \\
\text { datos podría conectarse a una instancia re- } \\
\text { mota de ASM y ambas en distintos centros de } \\
\text { datos, ocasionando lentitud en las transaccio- } \\
\text { nes generadas. } \\
\text { - Se requiere capacitación para la implementa- } \\
\text { ción y administración de Oracle RAC. }\end{array}$ & $\begin{array}{l}\text { - Con el tiempo la versión adquirida de Oracle, } \\
\text { tanto para la base de datos como para RAC, } \\
\text { perderá soporte. Mayor información en http:// } \\
\text { www.oracle.com/us/support/lifetime-sup- } \\
\text { port/index.html } \\
\text { - Nuevos errores (bugs) generados y no identifi- } \\
\text { cados por el soporte de Oracle a la fecha. }\end{array}$ \\
\hline
\end{tabular}

\section{Elaboración propia}




\section{Análisis de costo}

Se realizará un breve análisis económico basado en ciertos supuestos con el fin de dar un alcance general sobre la factibilidad de adquirir licencias de Oracle RAC y obtener un mayor nivel de disponibilidad, que se basará en la siguiente arquitectura:

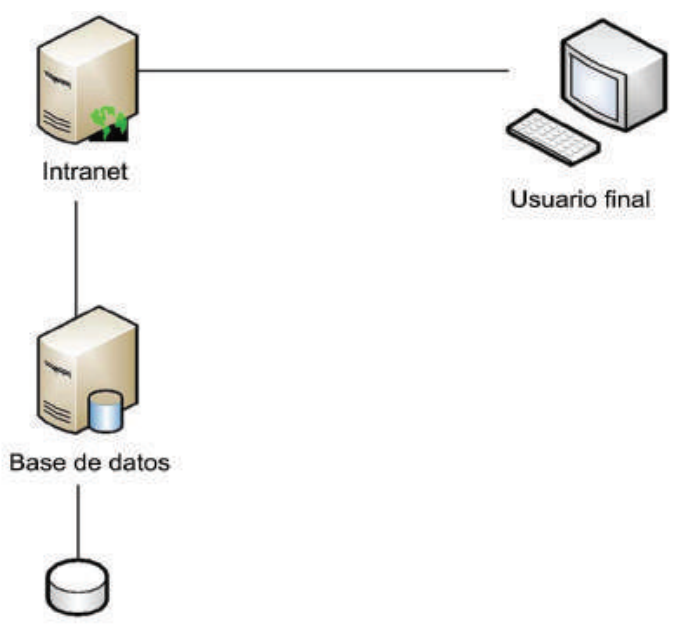

Figura 6. Arquitectura Single Instance Elaboración propia

Las consideraciones y premisas son las siguientes:

a) El servidor de Intranet y base de datos son servidores con soporte a procesadores de arquitectura Intel $\times 86-64$ y redundante en todos sus componentes físicos. Estos servidores cuentan con procesadores de 4 núcleos; el nivel de disponibilidad que ofrece cada uno de ellos es de 99,5\% anual.

b) El subsistema de almacenamiento es redundante en todos sus componentes físicos. El nivel de disponibilidad es de 99,9\% anual.

c) La comunicación SAN y LAN cuenta con dos equipos redundantes. Cada switch ofrece una disponibilidad de 99,9\% y 99,5\% anual, respectivamente.

d) Toda la infraestructura instalada se encuentra en un data center Tier II, con un nivel de disponibilidad de 99,75\% anual.

e) La base de datos es una versión Oracle Database 12c Enterprise Edition.

f) El costo por hora que pierde la empresa al no contar con la aplicación en funcionamiento es de $\$ 1100$. Se asume un costo de oportunidad de $10 \%$ en el proyecto de implementación de un Oracle Flex Cluster.

g) La empresa tiene personal con experiencia previa en implementación de clúster, además de contar con servidores libres con licencias de sistema operativo 
vigentes, por lo cual serán considerados como costos hundidos en el proyecto de implementación de Oracle Flex Cluster.

h) El impuesto general a la renta será de $30 \%$.

Basado en esta arquitectura básica, se define el nivel de disponibilidad respectivo:

- A: Disponibilidad del data center $=0.9975$

- B: Disponibilidad del subsistema de almacenamiento $=0.999$

- C: Disponibilidad de los switches SAN = $1-\left[(1-0.999)^{2}\right]=0.999999$

- D: Disponibilidad del servidor de base de datos $=0.995$

- E: Disponibilidad del servidor web $=0.995$

- F: Disponibilidad de los switches LAN $=1-\left[(1-0.995)^{2}\right]=0.999975$

Disponibilidad de la aplicación $=A \times B \times C \times D \times E \times F=0.98653674 \approx 98,654 \%$, la cual indica que el máximo tiempo de indisponibilidad que pudiera tener la aplicación es de aproximadamente 118 horas al año: (1 - 0.98653674)(24)(365) = 117.9381576.

A continuación se presenta una arquitectura con Oracle Flex Cluster, en alto nivel, para calcular el nivel de disponibilidad que ofrecería la aplicación del nuevo diseño:

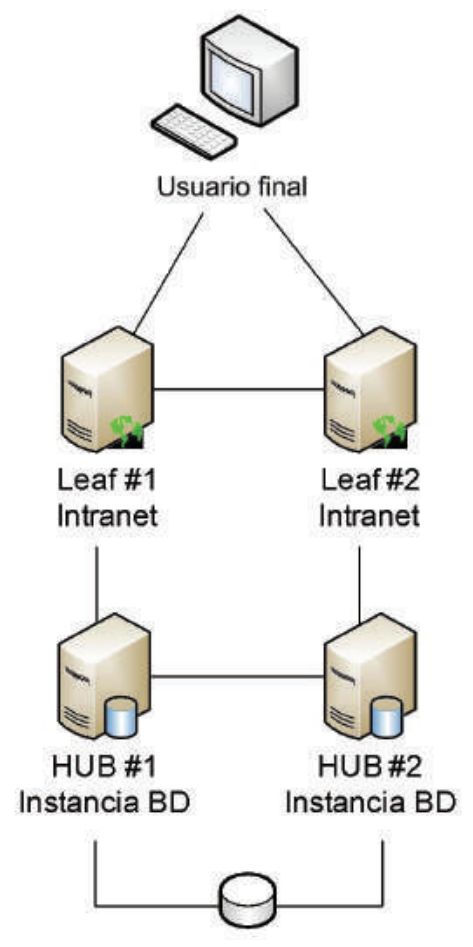

Figura 7. Arquitectura Oracle Flex Cluster Elaboración propia 
Nivel de disponibilidad de la aplicación:

- A: Disponibilidad del data center $=0.9975$

- B: Disponibilidad del subsistema de almacenamiento $=0.999$

- C: Disponibilidad de los switches SAN = $1-[(1-0.999) *(1-0.999)]=0.999999$

- D: Disponibilidad del servidor de base de datos $=1-\left[(1-0.995)^{2}\right]=0.999975$

- E: Disponibilidad del servidor web $==1-\left[(1-0.995)^{2}\right]=0.999975$

- F: Disponibilidad de los switches $L A N=1-\left[(1-0.995)^{2}\right]=0.999975$

Disponibilidad de la aplicación A x B X C x D x Ex F $=0.99642677 \approx 99,643 \%$, la cual indica que el máximo tiempo de indisponibilidad que pudiera tener la aplicación es de aproximadamente 32 horas al año: (1 - 0.99642677)(24)(365) = 31.30151448; la diferencia de la disponibilidad que ofrecen ambas soluciones es de $118-32=86$ horas.

Considerando el costo de $\$ 1100$ por hora, al no contar con el sistema disponible, la pérdida máxima por año sería de 86 horas $\times \$ 1100=\$ 94600$ anuales.

Para este breve análisis, los costos de las licencias Oracle que se presentan fueron realizados con base en la lista de precios publicados por Oracle (2015). De igual manera, el factor por multiplicar para el cálculo del número de licencias se encuentra disponible en la página web de Oracle (2009).

Los costos adicionales por adquirir las licencias de Oracle RAC se presentan en la tabla 5:

Tabla 5

Costos adicionales por adquirir las licencias de Oracle RAC

\begin{tabular}{|l|r|}
\hline \multicolumn{1}{|c|}{ Licencia } & Costo \\
\hline $\begin{array}{l}\text { Licencia de base de datos adicionales en el } \\
\text { nuevo servidor }\end{array}$ & 4 núcleos $* 0.5$ (factor) $=$ \\
$2 * \$ 47500=\$ 95000$
\end{tabular}

Elaboración propia

El flujo de caja del proyecto y los indicadores financieros correspondientes se presentan en las tablas 6 y 7. 
Tabla 6

Flujo de caja asociado al proyecto

\begin{tabular}{|c|c|c|c|c|c|c|}
\hline $\mathrm{CoK}=10 \%$ & Año 0 & Año 1 & Año 2 & Año 3 & Año 4 & Año 5 \\
\hline Inversión - licencias Oracle & $-\$ 224400$ & & & & & \\
\hline $\begin{array}{l}\text { Sistema indisponible - } \\
\text { máxima pérdida }\end{array}$ & & $\$ 94600$ & $\$ 94600$ & $\$ 94600$ & $\$ 94600$ & $\$ 94600$ \\
\hline Soporte anual & & $-\$ 44140$ & $-\$ 41140$ & $-\$ 41140$ & $-\$ 41140$ & $-\$ 41140$ \\
\hline $\begin{array}{l}\text { Amortización - licencias } \\
\text { Oracle }\end{array}$ & & $\$ 44880$ & $\$ 44880$ & $\$ 44880$ & $\$ 44880$ & $\$ 44880$ \\
\hline $\begin{array}{l}\text { Ahorro impuesto por } \\
\text { amortización (30 \%) }\end{array}$ & & $\$ 13464$ & $\$ 13464$ & $\$ 13464$ & $\$ 13464$ & $\$ 13464$ \\
\hline Flujo caja - proyecto & $-\$ 224400$ & $\$ 100604$ & $\$ 100604$ & $\$ 100604$ & $\$ 100604$ & $\$ 100604$ \\
\hline
\end{tabular}

\section{Elaboración propia}

Tabla 7

Indicadores financieros asociados al proyecto

\begin{tabular}{|l|c|}
\hline Indicador financiero & Valor \\
\hline Valor actual neto (VAN) & $\$ 17922,25$ \\
\hline Tasa interés de retorno (TIR) & $13 \%$ \\
\hline Indicador de beneficio/Costo sobre la inversión inicial (B/C) & 1.08 \\
\hline Período de retorno (Pay back) & 4 años \\
\hline
\end{tabular}

Elaboración propia

Basado en el escenario presentado y las premisas iniciales, la inversión de una implementación Oracle Flex Cluster es rentable. La nueva arquitectura proporcionará un nivel de disponibilidad a la aplicación de 99,643\%, comparado con el valor original de $98,654 \%$. Este análisis no considera otros beneficios adicionales que pueden obtenerse, como la escalabilidad, el balanceo de carga, etc.

\section{Conclusiones}

Con base en lo expuesto en el presente artículo, se brindan las siguientes conclusiones:

a) En el mundo agitado de hoy, donde la competitividad es clave para el éxito de las empresas, Oracle Flex Cluster ofrece una de las soluciones de alta disponibilidad 
más completas del mercado gracias a las nuevas tecnologías implementadas, lo que permite tener una disponibilidad de casi $100 \%$ y unos costos menores en comparación con otras soluciones.

b) Una vez comprendida la arquitectura y los aspectos técnicos que esta solución brinda, como se ha visto, la implementación de una solución de alto desempeño y escalabilidad, como las tecnologías de Oracle RAC y Flex Cluster, pueden ser fácilmente justificados económicamente.

c) La mejor opción de herramienta que se cuenta en la actualidad para la implementación de una base de datos de Oracle es Oracle Real Application Clusters, más conocida por sus siglas RAC; la cual hace posible la superación de las limitaciones de desempeño, así como las de escalabilidad, trabajando en un ambiente tradicional. Sus características permiten contar con una alta escalabilidad, al igual que con una alta disponibilidad, que son factores claves requeridos en las empresas para sus servicios de negocios críticos.

\section{Referencias}

Gómez, D. (2013). Oracle RAC 12c: Flex ASM y Flex Cluster. Oracle.com. Recuperado de http://www.oracle.com/technetwork/es/articles/database-performance/ caracteristicas-flex-asm-y-cluster-2088714-esa.html

Oracle. (2009). Oracle processor core factor table. Recuperado de http://www. oracle.com/us/corporate/contracts/processor-core-factor-table-070634.pdf

Oracle. (2015). Oracle technology global price list. Recuperado de http://www. oracle.com/us/corporate/pricing/technology-price-list-070617.pdf

Oracle Help Center. (2015). Database high availability overview. Recuperado de http://docs.oracle.com/database/121/HAOVW/architectures.htm\#CHDBIJAJ

Tpc.org. (2015). TPC-C V5. Recuperado de http://www.tpc.org/tpcc/

Womack, J. (2008). Oracle Database 11g: RAC Administration. Volume 1 - Student Guide. Recuperado de http://www.club-oracle.com/resources/oracle-11grac-student-guide-volume-1.1957/

\section{Glosario}

AAS (Average Active Sessions). Métrica sobre el número de sesiones que están trabajando o esperando en un intervalo de tiempo.

Off-premises. Hace referencia a un ambiente remoto, generalmente perteneciente a un tercero. 
On-premises: Hace referencia a un ambiente local, al cual generalmente se tiene acceso físico dado que es propio de la organización.

RPO (Recovery Time Objective). Tiempo máximo tolerable por falta de funcionamiento de las aplicaciones.

Round-Robin. Algoritmo por el cual se planifica la distribución equitativa de requerimientos.

TPC-C. Es un benchmark OLTP, que simula a un conjunto de usuarios, quienes ejecutan transacciones sobre la base de datos. 